“ (C) 2017 IEEE. Personal use of this material is permitted. Permission from IEEE must be obtained for all other uses, in any current or future media, including

reprinting/republishing this material for advertising or promotional purposes, creating new collective works, for resale or redistribution to servers or lists, or reuse of any copyrighted component of this work in other works." 


\section{Message from the IEEE TrustCom / BigDataSE / ICESS 2017 General Chairs}

As the General Chairs and on behalf of the Organizing Committee, it is our great pleasure to welcome you to the Joint 16th IEEE International Conference on Trust, Security and Privacy in Computing and Communications, 11th IEEE International Conference on Big Data Science and Engineering, and 14th IEEE International Conference On Embedded Software And Systems (IEEE TrustCom/BigDataSE/ICESS 2107), sponsored by IEEE and IEEE Computer Society, and endorsed by IEEE Technical Committee on Scalable Computing (TCSC).

We are pleased that TrustCom/BigDataSE/ICESS 2107 is held in Sydney this year. Sydney, capital of New South Wales and one of Australia's largest cities, is best known for its harbourfront Sydney Opera House, with a distinctive sail-like design. Massive Darling Harbour and the smaller Circular Quay port are hubs of waterside life, with the arched Harbour Bridge and esteemed Royal Botanic Garden nearby.

The technical program committee worked very hard to guarantee a successful conference. More than 300 papers were submitted to the main conferences and five different workshops and symposia organized in conjunction with the Conference. We wish to thank the authors of all submitted papers for choosing TrustCom/BigDataSE/ICESS 2107 as the forum to present their work. Highly qualified program committee members of each conference and workshop conducted a thorough review of the papers. The proceedings of the Conference will be published by the IEEE Computer Society Press (CPS) and IEEE Explore.

Many individuals have contributed to the success of this high-calibre international conference. We would like to express our special appreciation to the Steering Committee Chairs, Guojun Wang, Laurence T. Yang, Minyi Guo, and Jinjun Chen, for giving us the opportunity to hold this prestigious conference and for their guidance on the conference organization. We would also like to give our thanks to all the members of the Organizing Committee and Program Committee members for their effort and support.

We are grateful to all the authors who submitted their papers to the TrustCom/BigDataSE/ICESS 2107 conferences and workshops/symposia. We truly hope all the participants find the conference stimulating and constructive and at the same time enjoy their stay in Sydney.

Xiangjian He - University of Technology Sydney, Australia

Prasant Mohapatra - University of California, Davis, USA

Ravi Sandhu - University of Texas at San Antonio, USA

Song Guo - Hong Kong Polytechnic University, Hong Kong

Beniamino Di Martino - Second University of Naples, Italy

Jie Lu - University of Technology Sydney, Australia

Daniel Mosse - University of Pittsburgh, USA

Sudeep Pasricha - Colorado State University, USA

General Chairs of IEEE TrustCom/BigDataSE/ICESS 2017 